ROCKY MOUNTAIN

JOURNAL OF MATHEMATICS

Volume 39, Number 2, 2009

\title{
WAVELET TRANSFORM ON SPACES OF TYPE $W$
}

\author{
R.S. PATHAK AND GIREESH PANDEY
}

\begin{abstract}
The continuous wavelet transform is studied on certain Gelfand Shilov spaces of type $W$. The continuity and boundedness results for continuous wavelet transform are obtained on some suitably designed spaces of type $W$ defined on $\mathbf{R} \times \mathbf{R}_{+}, \mathbf{C} \times \mathbf{R}_{+}$and $\mathbf{C} \times \mathbf{C}$.
\end{abstract}

1. Introduction. The spaces of $W$-type were studied by Gelfand and Shilov [2]. They investigated the behavior of Fourier transformation on $W$-spaces. Also, $W$-spaces are applied to the theory of partial differential equations.

Pathak [4] and van Eijndhoven and Kerkhof [1] introduced new spaces of $W$-type and investigated the behavior of Hankel transformation over them.

The wavelet transform on Schwartz space $\mathcal{S}(\mathbf{R})$ and spaces of Sobolev type have been studied by many authors, see for example Holschneider [3]. In this paper, motivated by the work of Pathak [5] and Pathak and Upadhyay [6] we recall characterizations of $W$-type spaces introduced in [2] and study the behavior of continuous wavelet transform over them.

The continuous wavelet transform of a function $\phi$ with respect to the wavelet $\psi$ is defined by

$$
\left(\mathcal{W}_{\psi} \phi\right)(\sigma, a)=\widetilde{\Phi}(\sigma, a)=\int_{-\infty}^{\infty} \phi(t) \overline{\psi\left(\frac{t-\sigma}{a}\right)} \frac{d t}{a}
$$

provided the integral exists, where $a \in \mathbf{R}_{+}$and $\sigma \in \mathbf{R}$. If $\phi \in L^{2}(\mathbf{R})$ and $\psi \in L^{2}(\mathbf{R})$, then using the Parseval formula for Fourier transform,

2000 AMS Mathematics subject classification. Primary 42C40, 46F12, 46F15.

Keywords and phrases. $W$-type spaces, continuous wavelet transform.

The work of the second author was supported by CSIR (New Delhi), Grant No. 9/13(04)/2003/ EMR-I.

Received by the editors on June 13, 2006, and in revised form on September 7, 2006.

DOI:10.1216/RMJ-2009-39-2-619 Copyright (C)2009 Rocky Mountain Mathematics Consortium 
equation (1.1) can be rewritten in the form:

$$
\left(\mathcal{W}_{\psi} \phi\right)(\sigma, a)=\widetilde{\Phi}(\sigma, a)=\frac{1}{2 \pi} \int_{-\infty}^{\infty} e^{i \sigma x} \widehat{\phi}(x) \overline{\widehat{\psi}(a x)} d x
$$

where $\widehat{\phi}$ and $\widehat{\psi}$ denote Fourier transforms of $\phi$ and $\psi$, respectively.

2. The spaces $W_{M, \alpha}, W^{\Omega, \beta}$ and $W_{M, \alpha}^{\Omega, \beta}$. In this section we recall the definitions and properties of Gelfand-Shilov spaces of $W$-type. We also recall the behavior of Fourier transformation on the spaces $W_{M, \alpha}$, $W^{\Omega, \beta}$ and $W_{M, \alpha}^{\Omega, \beta}$ as given in [2].

Let $\mu(\xi), 0 \leq \xi<\infty$, and $\omega(\eta), 0 \leq \eta<\infty$, be continuous increasing functions such that $\mu(0)=0, \mu(\xi) \rightarrow \infty$ for $\xi \rightarrow \infty$ and $\omega(0)=0$, $\omega(\eta) \rightarrow \infty$ for $\eta \rightarrow \infty$. For $x \geq 0, y \geq 0$, we define

$$
M(x)=\int_{0}^{x} \mu(\xi) d \xi, \quad M(x)=M(-x) \quad \text { for } x<0
$$

and

$$
\Omega(y)=\int_{0}^{y} \omega(\eta) d \eta, \quad \Omega(y)=\Omega(-y) \quad \text { for } y<0 .
$$

The functions $M(x)$ and $\Omega(y)$ are continuous, increasing and convex with $M(0)=0, M(x) \rightarrow \infty$ for $x \rightarrow \infty$ and $\Omega(0)=0, \Omega(y) \rightarrow \infty$ for $y \rightarrow \infty$. We have the following fundamental convex inequalities

$$
M\left(x_{1}+x_{2}\right) \geq M\left(x_{1}\right)+M\left(x_{2}\right), \quad \Omega\left(y_{1}+y_{2}\right) \geq \Omega\left(y_{1}\right)+\Omega\left(y_{2}\right) .
$$

If the functions $\mu(\xi)$ and $\omega(\eta)$ are mutually inverse, that is, $\mu(\omega(\eta))=$ $\eta, \omega(\mu(\xi))=\xi$, then corresponding functions $M(x)$ and $\Omega(y)$ will be said to be dual in the sense of Young. In this case, the following Young inequality

$$
x y \leq M(x)+\Omega(y)
$$

holds for $x \geq 0, y \geq 0$.

Definition 2.1. The space $W_{M, \alpha}, \alpha>0$, consists of all complex valued infinitely differentiable functions $\phi(x),-\infty<x<\infty$, which for any $\delta>0$ satisfy 


$$
\left|\phi^{(q)}(x)\right| \leq C_{q \delta} e^{-M[(\alpha-\delta) x]}, \quad q=0,1,2, \ldots,
$$

where positive constants $C_{q \delta}$ depend on function $\phi(x)$.

Definition 2.2. The space $W^{\Omega, \beta}, \beta>0$, consists of all entire analytic functions $\phi(z), z=x+i y \in \mathbf{C}$, which for any $\rho>0$ satisfy

$$
\left|z^{k} \phi(z)\right| \leq C_{k \rho} e^{\Omega[(\beta+\rho) y]}, \quad k=0,1,2, \ldots,
$$

where positive constants $C_{k \rho}$ depend on function $\phi(z)$.

Definition 2.3. The space $W_{M, \alpha}^{\Omega, \beta}, \alpha>0, \beta>0$, consists of all entire analytic functions $\phi(z), z=x+i y \in \mathbf{C}$, which for any $\delta, \rho>0$ satisfy

$$
|\phi(z)| \leq C_{\delta \rho} e^{-M[(\alpha-\delta) x]+\Omega[(\beta+\rho) y]},
$$

where positive constants $C_{\delta \rho}$ depend on function $\phi(z)$.

The following properties are satisfied by the spaces $W_{M, \alpha}, W^{\Omega, \beta}$, $W_{M, \alpha}^{\Omega, \beta}[\mathbf{2}$, pages 12-24].

(1) The operation of differentiation is bounded in $W_{M, \alpha}, W^{\Omega, \beta}$, $W_{M, \alpha}^{\Omega, \beta}$, and hence is a continuous operation.

(2) The operation of multiplication by $x$ in $W_{M, \alpha}$ and multiplication by $z$ in $W^{\Omega, \beta}, W_{M, \alpha}^{\Omega, \beta}$ are bounded and hence are continuous operations.

Theorem 2.4. If the functions $M(x)$ and $\Omega(y)$ are mutually dual in the sense of Young, then the Fourier operator $\mathcal{F}: W_{M, \alpha} \rightarrow$ $W^{\Omega,(1 / \alpha)}, \mathcal{F}: W^{\Omega, \beta} \rightarrow W_{M,(1 / \beta)}$ is continuous and $\widehat{W}_{M, \alpha}=W^{\Omega,(1 / \alpha)}$, $\widehat{W}^{\Omega, \beta}=W_{M,(1 / \beta)}$.

Theorem 2.5. Let $\Omega_{1}(y)$ and $M_{1}(x)$ be the functions which are dual in the sense of Young to the functions $M(x)$ and $\Omega(y)$, respectively. Then the Fourier operator $\mathcal{F}: W_{M, \alpha}^{\Omega, \beta} \rightarrow W_{M_{1},(1 / \beta)}^{\Omega_{1},(1 / \alpha)}$ is continuous and $\widehat{W}_{M, \alpha}^{\Omega, \beta}=W_{M_{1},(1 / \beta)}^{\Omega_{1},(1 / \alpha)}$. 
In what follows we shall also need the following similar test function spaces, called spaces of type $\widetilde{W}$, which will be used in the study of the continuous wavelet transform.

Definition 2.6. The space $\widetilde{W}_{M, \alpha}$ is defined to be the set of all complex valued infinitely differentiable functions $\phi_{a}(\sigma)=\phi(\sigma, a) \in$ $C^{\infty}\left(\mathbf{R} \times \mathbf{R}_{+}\right)$which for any $\delta>0$ satisfy

$$
\begin{aligned}
&\left|\left(\frac{\partial}{\partial \sigma}\right)^{k}\left(\frac{\partial}{\partial a}\right)^{l} \phi(\sigma, a)\right| \leq C_{k l \delta} e^{-M[(\sigma / 1+a)(\alpha-\delta)]} \\
& k, l=0,1,2, \ldots
\end{aligned}
$$

where positive constants $C_{k l \delta}$ depend on the function $\phi$.

Definition 2.7. The space $\widetilde{W^{*}} \Omega, \Omega, \beta, a \beta$ is defined to be the set of all functions $\phi_{a}(s)=\phi(s, a) \in C^{\infty}\left(\mathbf{C} \times \mathbf{R}_{+}\right)$entirely analytic with respect to $s=\sigma+i \tau$ which, for any $\rho, \rho^{\prime}>0$, satisfy

$$
\begin{array}{rl}
\left|\left(\frac{s}{1+a}\right)^{k} \phi(s, a)\right| \leq C_{k \rho \rho^{\prime}} e^{\Omega[\tau(\beta+\rho)]+\Omega\left[\tau\left(a \beta+\rho^{\prime}\right)\right]} & \\
k & k=0,1,2, \ldots
\end{array}
$$

where positive constants $C_{k \rho \rho}$ depend on the function $\phi$.

Definition 2.8. The space $\widetilde{W}^{\Omega, \Omega, \beta, \beta}$ is defined to be the set of all functions $\phi(s, t) \in C^{\infty}(\mathbf{C} \times \mathbf{C})$ entirely analytic with respect to $s=\sigma+i \tau, t=a+i \gamma$, which, for any $\rho_{1}, \rho_{2}>0$, satisfy

$$
\begin{array}{r}
\left|\left(\frac{\partial}{\partial s}\right)^{k}\left(\frac{\partial}{\partial t}\right)^{l} \phi(s, t)\right| \leq C_{k l \rho_{1} \rho_{2}} e^{\Omega\left[\tau\left(\beta+\rho_{1}\right)\right]+\Omega\left[\gamma\left(\beta+\rho_{2}\right)\right]} ; \\
k, l=0,1,2, \ldots
\end{array}
$$

where positive constants $C_{k l \rho_{1} \rho_{2}}$ depend on the function $\phi$.

3. The wavelet transformation. In this section we study the wavelet transform (1.2) on spaces $W_{M, \alpha}, W^{\Omega, \beta}$ and $W_{M, \alpha}^{\Omega, \beta}$. 
Theorem 3.1. Let $M(x)$ and $\Omega(y)$ be the functions which are dual in the Young sense. Suppose $\widehat{\psi} \in W_{M, \alpha}$ and $\widehat{\phi} \in W_{M, \alpha}$. Then the wavelet transform $\Phi_{a}(s)=\widetilde{\Phi}(s, a) \in{\widetilde{W^{*}}}^{\Omega, \Omega,(1 / \alpha),(1 / a \alpha)}, s=\sigma+i \tau$ for arbitrary but fixed $a>0$, that is, wavelet transform $\left(\mathcal{W}_{\psi} \phi\right)(s, a)$ is a continuous linear map from $W^{\Omega, 1 / \alpha}$ into $\widetilde{W^{*}} \Omega, \Omega,(1 / \alpha),(1 / a \alpha)$.

Proof. The wavelet transform of a function $\phi$ with respect to the wavelet $\psi$ defined by (1.2) is

$$
\widetilde{\Phi}(\sigma, a)=\frac{1}{2 \pi} \int_{-\infty}^{\infty} e^{i \sigma x} \widehat{\phi}(x) \overline{\widehat{\psi}(a x)} d x
$$

Since $\widehat{\phi}, \widehat{\psi} \in W_{M, \alpha}$, therefore, the wavelet transform can be extended to the complex values of $s=\sigma+i \tau$ according to the definition

$$
\begin{aligned}
\tilde{\Phi}(\sigma+i \tau, a) & =\frac{1}{2 \pi} \int_{-\infty}^{\infty} e^{i(\sigma+i \tau) x} \widehat{\phi}(x) \overline{\widehat{\psi}(a x)} d x \\
& =\frac{1}{2 \pi} \int_{-\infty}^{\infty} e^{i s x} \widehat{\phi}(x) \overline{\widehat{\psi}(a x)} d x
\end{aligned}
$$

Integrating by parts $k$ times, we get

$$
\begin{aligned}
\left|(i s)^{k} \tilde{\Phi}(s, a)\right| & =\left|\frac{(-1)^{k}}{2 \pi} \int_{-\infty}^{\infty} e^{i s x} D_{x}^{k}(\widehat{\phi}(x) \overline{\widehat{\psi}(a x)}) d x\right| \\
& =\left|\frac{(-1)^{k}}{2 \pi} \sum_{l=0}^{k}\left(\begin{array}{c}
k \\
l
\end{array}\right) \int_{-\infty}^{\infty} e^{i s x} D_{x}^{(k-l)} \widehat{\phi}(x) D_{x}^{(l)} \overline{\widehat{\psi}(a x)} d x\right| \\
& =\left|\frac{(-1)^{k}}{2 \pi} \sum_{l=0}^{k}\left(\begin{array}{l}
k \\
l
\end{array}\right) \int_{-\infty}^{\infty} e^{i s x} \widehat{\phi}^{(k-l)}(x) a^{l} \widehat{\hat{\psi}}^{(l)}(a x) d x\right| \\
& \leq \frac{1}{2 \pi}(1+a)^{k} \sum_{l=0}^{k}\left(\begin{array}{c}
k \\
l
\end{array}\right) \int_{-\infty}^{\infty}\left|e^{i s x} \widehat{\phi}^{(k-l)}(x) \overline{\widehat{\psi}^{(l)}(a x)}\right| d x
\end{aligned}
$$


Now using Definition 2.1, we get

$$
\begin{aligned}
& |I|=\left|\left(\frac{s}{1+a}\right)^{k} \widetilde{\Phi}(s, a)\right| \\
& \leq \frac{1}{2 \pi} \sum_{l=0}^{k}\left(\begin{array}{l}
k \\
l
\end{array}\right) \int_{-\infty}^{\infty}\left|e^{i s x}\right|\left|\widehat{\phi}^{(k-l)}(x)\right|\left|\overline{\widehat{\psi}^{(l)}(a x)}\right| d x \\
& \leq \frac{1}{2 \pi} \sum_{l=0}^{k}\left(\begin{array}{l}
k \\
l
\end{array}\right) \int_{-\infty}^{\infty} e^{-\tau x} C_{(k-l) \delta} e^{-M[x(\alpha-\delta)]} C_{l \delta^{\prime}} e^{-M\left[a x\left(\alpha-\delta^{\prime}\right)\right]} d x \\
& \leq \frac{1}{2 \pi} \sum_{l=0}^{k}\left(\begin{array}{l}
k \\
l
\end{array}\right) C_{(k-l) \delta} C_{l \delta^{\prime}} \int_{-\infty}^{\infty} e^{2|\tau x|-M[x(\alpha-\delta)]-M\left[a x\left(\alpha-\delta^{\prime}\right)\right]} d x \\
& =C_{k \delta \delta^{\prime}}^{\prime} \int_{-\infty}^{\infty} e^{2|\tau x|-M[x(\alpha-\delta)]-M\left[a x\left(\alpha-\delta^{\prime}\right)\right]} d x
\end{aligned}
$$

where $C_{k \delta \delta^{\prime}}^{\prime}=1 /(2 \pi) \sum_{l=0}^{k}\left(\begin{array}{l}k \\ l\end{array}\right) C_{(k-l) \delta} C_{l \delta^{\prime}}$. Now, applying (2.3) and Young's inequality (2.4), the exponent in the above integral can be transformed as follows:

$$
\begin{aligned}
-M[x(\alpha-\delta)]+|\tau x| & \leq-M[x(\alpha-\delta)]+M[x(\alpha-2 \delta)]+\Omega\left[\frac{\tau}{\alpha-2 \delta}\right] \\
& \leq-M[\delta x]+\Omega\left[\frac{\tau}{\alpha-2 \delta}\right]
\end{aligned}
$$

and

$$
\begin{aligned}
-M\left[a x\left(\alpha-\delta^{\prime}\right)\right]+|\tau x| & \\
& \leq-M\left[a x\left(\alpha-\delta^{\prime}\right)\right]+M\left[a x\left(\alpha-2 \delta^{\prime}\right)\right]+\Omega\left[\frac{\tau}{a\left(\alpha-2 \delta^{\prime}\right)}\right] \\
& \leq-M\left[\delta^{\prime} a x\right]+\Omega\left[\frac{\tau}{a\left(\alpha-2 \delta^{\prime}\right)}\right]
\end{aligned}
$$

Therefore, we get the estimate

$$
\begin{aligned}
|I| & \leq C_{k \delta \delta^{\prime}}^{\prime} e^{\Omega[\tau /(\alpha-2 \delta)]+\Omega\left[\tau /\left(a\left(\alpha-2 \delta^{\prime}\right)\right)\right]} \int_{-\infty}^{\infty} e^{-M[\delta x]-M\left[\delta^{\prime} a x\right]} d x \\
& \leq C_{k \rho \rho^{\prime}}^{\prime \prime} e^{\Omega[\tau((1 / \alpha)+\rho)]+\Omega\left[\tau\left((1 / a \alpha)+\rho^{\prime}\right)\right]}
\end{aligned}
$$


In the above, we set $1 /(\alpha-2 \delta)=(1 / \alpha)+\rho$ and $1 /\left(a\left(\alpha-2 \delta^{\prime}\right)\right)=$ $(1 / a \alpha)+\rho^{\prime}$ where the quantities $\rho$ and $\rho^{\prime}$ are arbitrarily small together with $\delta$ and $\delta^{\prime}$. Thus, $\widetilde{\Phi}(s, a) \in \widetilde{W^{*}} \Omega, \Omega,(1 / \alpha),(1 / a \alpha)$.

Theorem 3.2. Let the functions $M(x)$ and $\Omega(y)$ be the same as in Theorem 3.1. Suppose that $\widehat{\psi} \in W^{\Omega, \beta}$ and $\widehat{\phi} \in W^{\Omega, \beta}$. Then the wavelet transform $\left(\mathcal{W}_{\psi} \phi\right)(\sigma, a)$ is a continuous linear map from $W_{M, 1 / \beta}$ into $\widetilde{W}_{M, 1 / \beta}$, that is, $\widetilde{\Phi}(\sigma, a) \in \widetilde{W}_{M, 1 / \beta}$.

Proof. Since $\widehat{\phi} \in W^{\Omega, \beta}$, following the technique of [2, page 22], the expression for the wavelet transform defined by (1.2) can be written as

$$
\begin{aligned}
\widetilde{\Phi}(\sigma, a) & =\frac{1}{2 \pi} \int_{-\infty}^{\infty} e^{i \sigma(x+i y)} \widehat{\phi}(x+i y) \overline{\hat{\psi}(a(x+i y))} d x \\
& =\frac{1}{2 \pi} \int_{-\infty}^{\infty} e^{i \sigma z} \widehat{\phi}(z) \overline{\widehat{\psi}(a z)} d x, \quad z=x+i y .
\end{aligned}
$$

For nonnegative integers $k$ and $l$, after differentiation of (3.2), we get

$$
\begin{aligned}
\left(\frac{\partial}{\partial \sigma}\right)^{k}\left(\frac{\partial}{\partial a}\right)^{l} \widetilde{\Phi}(\sigma, a) & =\frac{1}{2 \pi} \int_{-\infty}^{\infty} e^{i \sigma z}(i z)^{k} \widehat{\phi}(z)\left(\frac{\partial}{\partial a}\right)^{l} \overline{\widehat{\psi}(a z)} d x \\
& =\frac{1}{2 \pi} \int_{-\infty}^{\infty} e^{i \sigma z}(i z)^{k} \widehat{\phi}(z) z^{l}\left(\frac{\partial}{\partial(a z)}\right)^{l} \overline{\hat{\psi}(a z)} d x \\
& =\frac{i^{k}}{2 \pi} \int_{-\infty}^{\infty} e^{i \sigma z} z^{k+l} \widehat{\phi}(z) \overline{\hat{\psi}^{(l)}(a z)} d x
\end{aligned}
$$

Now, using the inequality $|z|^{l} \leq\left(|z|^{l+2}+|z|^{l}\right) /\left(x^{2}+1\right)$ and conditions for including $\widehat{\phi}, \widehat{\psi}$ in $W^{\Omega, \beta}$, we obtain

$$
\begin{aligned}
|I| & =\left|\left(\frac{\partial}{\partial \sigma}\right)^{k}\left(\frac{\partial}{\partial a}\right)^{l} \widetilde{\Phi}(\sigma, a)\right| \\
& \leq \frac{1}{2 \pi} \int_{-\infty}^{\infty}\left|e^{i \sigma z}\right|\left(\frac{|z|^{k+l+2}+|z|^{k+l}}{x^{2}+1}\right)|\widehat{\phi}(z)|\left|\overline{\widehat{\psi}^{(l)}(a z)}\right| d x \\
& \leq \frac{1}{2 \pi} \int_{-\infty}^{\infty} e^{-\sigma y}\left[C_{k+l+2, \rho}+C_{k+l, \rho}\right] e^{\Omega[y(\beta+\rho)]}
\end{aligned}
$$




$$
\begin{aligned}
& \qquad \times C_{0 \rho^{\prime} l} e^{\Omega\left[a y\left(\beta+\rho^{\prime}\right)\right]} \frac{d x}{x^{2}+1} \\
& \leq C_{k l \rho \rho^{\prime}}^{\prime} e^{-\sigma y+\Omega[y(\beta+\rho)]+\Omega\left[a y\left(\beta+\rho^{\prime}\right)\right]} \int_{-\infty}^{\infty} \frac{d x}{x^{2}+1} \\
& \leq C_{k l \rho \rho^{\prime}}^{\prime \prime} e^{-\sigma y+\Omega\left[y\left(\beta+\rho+a\left(\beta+\rho^{\prime}\right)\right)\right]}, \text { for all } \rho, \rho^{\prime}>0 \\
& \leq C_{k l \rho \rho^{\prime}}^{\prime \prime} e^{-\sigma y+\Omega[y(1+a)(\beta+\rho)]}, \text { for } \rho=\rho^{\prime} .
\end{aligned}
$$

Until now $y$ has been an arbitrary number. Using the technique [2, page 22], let us now choose the sign of $y$ in such a manner that the equality $\sigma y=|\sigma||y|$ and the absolute value of $y$ are satisfied so that the Young inequality (2.4) becomes equality

$$
|\sigma||y|=\Omega[|y|(1+a)(\beta+\rho)]+M\left[\frac{|\sigma|}{(1+a)(\beta+\rho)}\right] .
$$

Then, the exponent in expression (3.3) becomes

$$
-\sigma y+\Omega[y(1+a)(\beta+\rho)]=-M\left[\frac{|\sigma|}{(1+a)(\beta+\rho)}\right] .
$$

Replacing $1 /(\beta+\rho)$ by $(1 / \beta)-\delta$ where $\delta$ is arbitrarily small, we obtain the estimate for the expression (3.3)

$$
\left|\left(\frac{\partial}{\partial \sigma}\right)^{k}\left(\frac{\partial}{\partial a}\right)^{l} \widetilde{\Phi}(\sigma, a)\right| \leq C_{k l \delta}^{\prime \prime} e^{-M[|\sigma| /(1+a)((1 / \beta)-\delta)]} .
$$

Hence, wavelet transform $\widetilde{\Phi}(\sigma, a) \in \widetilde{W}_{M, 1 / \beta}$.

Theorem 3.3. Let functions $M(x)$ and $\Omega(y)$ be the same as in Theorem 3.1. Suppose $\widehat{\psi} \in W^{\Omega,(1 / \beta)}$ and $\widehat{\phi} \in W_{M, \beta}$. Then the wavelet transform $\left(\mathcal{W}_{\psi} \phi\right)(s, t)$ extends to an entire function of $s=\sigma+i \tau$, $t=a+i \gamma$ and it is a continuous linear map from $W^{\Omega,(1 / \beta)}$ into $\widetilde{W}^{\Omega, \Omega,(1 / \beta),(1 / \beta)}$.

Proof. The wavelet transform of a function $\phi$ with respect to the wavelet $\psi$ defined by (1.2) is

$$
\begin{aligned}
\tilde{\Phi}(\sigma, a) & =\frac{1}{2 \pi} \int_{-\infty}^{\infty} e^{i \sigma x} \widehat{\phi}(x) \overline{\widehat{\psi}(a x)} d x \\
& =\frac{1}{2 \pi} \int_{-\infty}^{\infty} e^{i \sigma x} \widehat{\phi}(x) \int_{-\infty}^{\infty} e^{i \xi a x} \overline{\psi(\xi)} d \xi d x .
\end{aligned}
$$


Since both $\widehat{\phi}$ and $\psi \in W_{M, \beta}$, from [2, page 20] we have

$$
\widetilde{\Phi}(\sigma+i \tau, a+i \gamma)=\frac{1}{2 \pi} \int_{-\infty}^{\infty} e^{i(\sigma+i \tau) x} \widehat{\phi}(x) \int_{-\infty}^{\infty} e^{i \xi(a+i \gamma) x} \overline{\psi(\xi)} d \xi d x
$$

so that

$$
\widetilde{\Phi}(s, t)=\frac{1}{2 \pi} \int_{-\infty}^{\infty} e^{i s x} \widehat{\phi}(x) \int_{-\infty}^{\infty} e^{i \xi t x} \overline{\psi(\xi)} d \xi d x
$$

For $k, l \in \mathbf{N}_{0}$, after differentiation of (3.4),we get

$$
\begin{aligned}
\left(\frac{\partial}{\partial t}\right)^{k}\left(\frac{\partial}{\partial s}\right)^{l} \widetilde{\Phi}(s, t)= & \frac{1}{2 \pi} \int_{-\infty}^{\infty} e^{i s x}(i x)^{l} \widehat{\phi}(x) \\
& \times \int_{-\infty}^{\infty} e^{i \xi t x}(i x \xi)^{k} \overline{\psi(\xi)} d \xi d x \\
= & \frac{1}{2 \pi} \int_{-\infty}^{\infty} e^{i s x}(i x)^{l+k} \widehat{\phi}(x) \\
& \times \int_{-\infty}^{\infty} e^{i \xi t x} \xi^{k} \overline{\psi(\xi)} d \xi d x .
\end{aligned}
$$

Now, using the definitions for $\widehat{\phi}$ and $\psi$, we obtain

$$
\begin{aligned}
|I|= & \left|\left(\frac{\partial}{\partial t}\right)^{k}\left(\frac{\partial}{\partial s}\right)^{l} \widetilde{\Phi}(s, t)\right| \\
\leq & \frac{1}{2 \pi} \int_{-\infty}^{\infty}\left|e^{i s x}\right|\left|(i x)^{l+k} \widehat{\phi}(x)\right| \\
& \times \int_{-\infty}^{\infty}\left|e^{i \xi t x}\right|\left|\xi^{k} \overline{\psi(\xi)}\right| d \xi d x \\
\leq & \frac{1}{2 \pi} \int_{-\infty}^{\infty} e^{-\tau x} C_{0 k l \delta} e^{-M[x(\beta-\delta)]} \\
& \times \int_{-\infty}^{\infty} e^{-\gamma x \xi} C_{0 k \delta^{\prime}} e^{-M\left[\xi\left(\beta-\delta^{\prime}\right)\right]} d \xi d x \\
\leq & \frac{1}{2 \pi} C_{0 k l \delta} C_{0 k \delta^{\prime}} \int_{-\infty}^{\infty} e^{|\tau x|-M[x(\beta-\delta)]} \\
& \times \int_{-\infty}^{\infty} e^{|\gamma x \xi|-M\left[\xi\left(\beta-\delta^{\prime}\right)\right]} d \xi d x
\end{aligned}
$$


Applying Young's inequality (2.4) in the exponent (3.5), we get

$$
\begin{aligned}
|\tau x|-M[x(\beta-\delta)] & \leq \Omega\left[\frac{|\tau|}{\beta-2 \delta}\right]+M[|x|(\beta-2 \delta)]-M[x(\beta-\delta)] \\
& \leq \Omega\left[\frac{\tau}{\beta-2 \delta}\right]-M[\delta x]
\end{aligned}
$$

and

$$
\begin{aligned}
|\gamma x \xi|-M\left[\xi\left(\beta-\delta^{\prime}\right)\right] & \leq \Omega\left[\frac{|\gamma|}{\beta-2 \delta^{\prime}}\right]+M\left[|x \xi|\left(\beta-2 \delta^{\prime}\right)\right]-M\left[\xi\left(\beta-\delta^{\prime}\right)\right] \\
& \leq \Omega\left[\frac{\gamma}{\beta-2 \delta^{\prime}}\right]-M\left[\xi\left(\left(\beta-\delta^{\prime}\right)-x\left(\beta-2 \delta^{\prime}\right)\right)\right] .
\end{aligned}
$$

Setting $1 /(\beta-2 \delta)=(1 / \beta)+\rho$ and $1 /\left(\beta-2 \delta^{\prime}\right)=(1 / \beta)+\rho^{\prime}$ where $\rho$ and $\rho^{\prime}$ are arbitrarily small together with $\delta$ and $\delta^{\prime}$, expression (3.5) becomes

$$
\begin{aligned}
|I| \leq & \frac{1}{2 \pi} C_{0 k l \delta} C_{0 k \delta^{\prime}} e^{\Omega[\tau /(\beta-2 \delta)]} e^{\Omega\left[\gamma /\left(\beta-2 \delta^{\prime}\right)\right]} \\
& \times \int_{-\infty}^{\infty} e^{-M[\delta x]} \int_{-\infty}^{\infty} e^{-M\left[\xi\left(\left(\beta-\delta^{\prime}\right)-x\left(\beta-2 \delta^{\prime}\right)\right)\right]} d \xi d x \\
\leq & C_{k l \rho \rho^{\prime}}^{\prime} e^{\Omega[\tau((1 / \beta)+\rho)]} e^{\Omega\left[\gamma\left((1 / \beta)+\rho^{\prime}\right)\right]} I_{1},
\end{aligned}
$$

where $I_{1}$ denotes the double integral which is estimated as follows:

$$
\begin{aligned}
I_{1} & =\int_{-\infty}^{\infty} e^{-M[\delta x]} \int_{-\infty}^{\infty} e^{-M\left[\xi\left(\left(\beta-\delta^{\prime}\right)-x\left(\beta-2 \delta^{\prime}\right)\right)\right]} d \xi d x \\
& =\int_{-\infty}^{\infty} e^{-M[\delta x]} \int_{-\infty}^{\infty} \frac{e^{-M[u]}}{\left(\beta-\delta^{\prime}\right)-\left(\beta-2 \delta^{\prime}\right) x} d u d x \\
& =\int_{-\infty}^{\infty} e^{-M[u]} d u \int_{-\infty}^{\infty} \frac{e^{-M[\delta x]}}{\left(\beta-\delta^{\prime}\right)-\left(\beta-2 \delta^{\prime}\right) x} d x \\
& =\frac{A}{\beta-2 \delta^{\prime}} \int_{-\infty}^{\infty} \frac{e^{-M[\delta x]}}{\theta-x} d x,
\end{aligned}
$$

where

$$
\theta=\frac{\beta-\delta^{\prime}}{\beta-2 \delta^{\prime}}
$$


and

$$
A=\int_{-\infty}^{\infty} e^{-M[u]} d u, \quad I_{1}=\frac{A}{\beta-2 \delta^{\prime}} \mathcal{H}\left(e^{-M[\delta x]}\right)(\theta)
$$

where $\mathcal{H}$ denotes the Hilbert transform. Since $e^{-M[\delta x]} \in L^{p}(\mathbf{R})$ for $p>1$, from [7, page 275], we have

$$
\left\|\mathcal{H}\left(e^{-M[\delta x]}\right)(\theta)\right\|_{p} \leq C_{p}\left\|e^{-M[\delta x]}\right\|_{p}
$$

Since the same $L^{p}$ estimate is valid for first order derivatives, from the Sobolev embedding theorem, we have that the Hilbert transform $\mathcal{H}\left(e^{-M[\delta x]}\right)(\theta)$ belongs to $L^{\infty}$. Hence,

$$
\left|I_{1}\right|<\infty \text {. }
$$

Consequently, it follows from (3.6) that $\widetilde{\Phi}(s, t) \in \widetilde{W}^{\Omega, \Omega,(1 / \beta),(1 / \beta)}$.

Theorem 3.4. Let $\Omega_{1}(y)$ and $M_{1}(x)$ be the functions which are dual in the sense of Young to the functions $M(x)$ and $\Omega(y)$, respectively. Suppose $\psi \in W_{M_{1}, \beta}$ and $\widehat{\phi} \in W_{M, \alpha}^{\Omega, \beta}$. Then, for a fixed $a \in \mathbf{R}_{+}$, the wavelet transform $\widetilde{\Phi}(s, a)$ as a function of $s=\sigma+i \tau$ belongs to $W_{M_{1},(1 / \lambda)}^{\Omega_{1},(1 / \alpha)}$ with $\lambda=\beta+a / \beta$.

Proof. By [2, page 24], the expression for the wavelet transform of the function $\widehat{\phi} \in W_{M, \alpha}^{\Omega, \beta}$ can be written as

$$
\begin{aligned}
\tilde{\Phi}(\sigma+i \tau, a)= & \frac{1}{2 \pi} \int_{-\infty}^{\infty} e^{i(\sigma+i \tau)(x+i y)} \widehat{\phi}(x+i y) \\
& \times \int_{-\infty}^{\infty} e^{i \xi a(x+i y)} \overline{\psi(\xi)} d \xi d x \\
\tilde{\Phi}(s, a)= & \frac{1}{2 \pi} \int_{-\infty}^{\infty} e^{i s z} \widehat{\phi}(x+i y) \int_{-\infty}^{\infty} e^{i \xi a z} \overline{\psi(\xi)} d \xi d x
\end{aligned}
$$

where $s=\sigma+i \tau$ and $z=x+i y$. Carrying out the transformations used in Theorems 3.1 and 3.2, an estimate of the absolute value of (3.7) yields 


$$
\begin{aligned}
& |\tilde{\Phi}(s, a)| \leq \frac{1}{2 \pi} \int_{-\infty}^{\infty}\left|e^{i s z}\right||\widehat{\phi}(z)| \int_{-\infty}^{\infty}\left|e^{i \xi a z}\right||\overline{\psi(\xi)}| d \xi d x \\
& \leq \frac{1}{2 \pi} \int_{-\infty}^{\infty} e^{-\tau x-\sigma y} C_{\delta \rho} e^{-M[(\alpha-\delta) x]+\Omega[(\beta+\rho) y]} \\
& \times \int_{-\infty}^{\infty} e^{-a y \xi} C_{0 \delta^{\prime}} e^{-M_{1}\left[\left(\beta-\delta^{\prime}\right) \xi\right]} d \xi d x \\
& \leq \frac{1}{2 \pi} C_{\delta \rho} C_{0 \delta^{\prime}} e^{-\sigma y+\Omega[(\beta+\rho) y]} \int_{-\infty}^{\infty} e^{|\tau x|-M[(\alpha-\delta) x]} \\
& \times \int_{-\infty}^{\infty} e^{|a y \xi|-M_{1}\left[\left(\beta-\delta^{\prime}\right) \xi\right]} d \xi d x \\
& \leq \frac{1}{2 \pi} C_{\delta \rho} C_{0 \delta^{\prime}} e^{-\sigma y+\Omega[(\beta+\rho) y]} e^{\Omega_{1}[\tau /(\alpha-2 \delta)]} e^{\Omega\left[(a y) /\left(\beta-2 \delta^{\prime}\right)\right]} \\
& \times \int_{-\infty}^{\infty} e^{-M[\delta x]} \int_{-\infty}^{\infty} e^{-M_{1}\left[\delta^{\prime} \xi\right]} d \xi d x \\
& \leq C_{\delta \delta^{\prime} \rho}^{\prime} e^{-\sigma y+\Omega\left[\left(\beta+\rho+\left(a / \beta-2 \delta^{\prime}\right)\right) y\right]} e^{\Omega_{1}[\tau /(\alpha-2 \delta)]} \\
& \leq C_{\rho \rho^{\prime} \rho^{\prime \prime}}^{\prime} e^{-\sigma y+\Omega\left[\left(\beta+\rho+(a / \beta)+\rho^{\prime \prime}\right) y\right]} e^{\Omega_{1}\left[\tau\left((1 / \alpha)+\rho^{\prime}\right)\right]} \\
& \text { (on setting } \frac{a}{\beta-2 \delta^{\prime}}=\frac{a}{\beta}+\rho^{\prime \prime}, \quad \frac{1}{\alpha-2 \delta}=\frac{1}{\alpha}+\rho^{\prime} \text { ) } \\
& \leq C_{\rho \rho^{\prime} \rho^{\prime \prime \prime}}^{\prime} e^{-\sigma y+\Omega\left[\left(\beta+(a / \beta)+\rho^{\prime \prime \prime}\right) y\right]} e^{\Omega_{1}\left[\tau\left(1 /\left(\alpha+\rho^{\prime}\right)\right)\right]} ; \\
& \text { where } \rho+\rho^{\prime \prime}=\rho^{\prime \prime \prime} \\
& =C_{\rho \rho^{\prime} \rho^{\prime \prime \prime}}^{\prime} e^{-M_{1}\left[\sigma /\left(\beta+(a / \beta)+\rho^{\prime \prime \prime}\right)\right]} e^{\Omega_{1}\left[\tau\left((1 / \alpha)+\rho^{\prime}\right)\right]} \\
& =C_{\rho \rho^{\prime} \rho^{\prime \prime \prime}}^{\prime} e^{-M_{1}\left[\sigma /\left(\lambda+\rho^{\prime \prime \prime}\right)\right]} e^{\Omega_{1}\left[\tau\left((1 / \alpha)+\rho^{\prime}\right)\right]} ; \\
& \text { where } \lambda=\beta+\frac{a}{\beta} \\
& =C_{\delta^{\prime \prime} \rho^{\prime}}^{\prime} e^{-M_{1}\left[\sigma\left((1 / \lambda)-\delta^{\prime \prime}\right)\right]} e^{\Omega_{1}\left[\tau\left((1 / \alpha)+\rho^{\prime}\right)\right]} ; \\
& \text { where } \frac{1}{\lambda+\rho^{\prime \prime \prime}}=\frac{1}{\lambda}-\delta^{\prime \prime} \text {. }
\end{aligned}
$$

Thus, $\widetilde{\Phi}(s, a) \in W_{M_{1},(1 / \lambda)}^{\Omega_{1},(1 / \alpha)}, \lambda=\beta+(a / \beta)$.

Acknowledgments. The authors express their sincere thanks to the referee who suggested several improvements in the original version of the manuscript. 


\section{REFERENCES}

1. S.J.L. van Eijndhoven and M.J. Kerkhof, The Hankel transformation and spaces of type $W$, in Reports on applied and numerical analysis, Department of Mathematics and Computing Science, Eindhoven University of Technology, 1988.

2. I.M. Gelfand and G.E. Shilov, Generalized functions, Vol. III, Academic Press, New York, 1967.

3. M. Holschneider, Wavelets: An analysis tool, Clarendon Press, Oxford, 1995.

4. R.S. Pathak, On Hankel transformable spaces and a Cauchy problem, Canad. J. Math. 37 (1985), 84-106.

5. — Wavelet transform of distributions, Tohoku Math. J. 56 (2004), 411-421.

6. R.S. Pathak and S.K. Upadhyay, $W^{p}$-spaces and Fourier transform, Proc. Amer. Math. Soc. 121 (1994), 733-738.

7. A.I. Zayed, Handbook of function and generalized function transformations, CRC Press, New York, 1996.

Department of Mathematics, Banaras Hindu University, Varanasi-221 005, INDIA

Email address: ramshankarpathak@yahoo.co.in

Department of Mathematics, Banaras Hindu University, Varanasi-221 005, INDIA

Email address: gp2_bhu@yahoo.com 